\title{
ANCLAJES POSINSTALADOS EN CONCRETOS DE ALTA RESISTENCIA
}

\section{ANCHORS POST-INSTALLED IN HIGH STRENGTH CONCRETE}

\author{
Yuber Mauricio Ruda Arias* \\ Diego Fernando Páez Moreno*
}

Fecha de recepción: 2 de septiembre de 2016

Fecha de revisión: 3 de febrero de 2017

Fecha de aprobación: 13 de marzo de 2017

Cómo citar: Y. M. Ruda Arias y D. F. Páez Moreno, "Anclajes posinstalados en concretos de alta resistencia," Ciencia e Ingeniería Neogranadina, vol. 27, no. 2, pp. 137-156. DOI: http://dx.doi.org/10.18359/rcin.2208

\section{RESUMEN}

Hoy en día, las grandes construcciones de Colombia y el mundo, y en especial las de infraestructura vial, han fomentado el uso de concretos de alta resistencia; también ha crecido de forma simultánea el uso de los anclajes posinstalados en todas las estructuras de concreto. Por lo anterior, se desarrolló en esta investigación un procedimiento experimental para evaluar sistemas de anclaje posinstalados, basado en ensayos a tracción con probetas de concreto de alta resistencia. La evaluación considera el análisis de los resultados de los ensayos a tracción de probetas con un sistema de anclaje para tres longitudes $(6,9$ y 12 veces el diámetro de la varilla) con dos diámetros de varilla (3/8" y $5 / 8^{\prime \prime}$ ) en dos calidades de concreto de alta resistencia (5000Psi y 6000Psi), para determinar la longitud y carga efectiva del sistema. El análisis que se realiza al ensayo experimental es correlacionar el resultado de la carga máxima de fluencia del acero de refuerzo utilizado (fy=4200Psi) con las variables del sistema de anclaje posinstalado. Con esto se obtuvo como resultados el porcentaje de resistencia del sistema en función de la longitud de anclaje, la longitud efectiva que garantiza la fluencia del acero y las

\footnotetext{
* Ingeniero de Transporte y Vias; Ingeniero de Diseño Esrtuctural; candidato a magíster en Ingenieria con enfasis en Infraetructura Vial. Correo electrónico: yuber.ruda@uptc.edu.co. ORCID: http://orcid.org/0000-0002-9519-1609

** Ingeniero Civil, de la Universidad Nacional de Colombia; magíster en Ingeniería Civil, de a Universidad de los Andes, Profesor de planta, de la Universidad Pedagógica y Tecnológica de Colombia, Tunja, Colombia. Correo electrónico: diego.paez@uptc.edu.co. ORCID: http://orcid.org/0000-0003-1141-8819
} 
correlaciones entre el porcentaje de resistencia efectiva alcanzada para cada longitud de anclaje con el uso de concreto de alta resistencia. Con los anteriores parámetros se analizó el aporte de los concretos de alta resistencia a la eficiencia de los sistemas de anclajes posinstalados.

Palabras clave: Anclaje posinstalado, concreto de alta resistencia.

\section{ABSTRACT}

Nowadays the great constructions of Colombia and the world, especially road infrastructure, have encouraged the use of high-strength concrete. The use of post-installed anchors has also grown simultaneously in all concrete structures. Therefore an experimental procedure was developed in this investigation to assess post-installed anchor systems based on tensile test specimens with high strength concrete. The evaluation considers the analysis of the tensile tests results of test pieces with an anchoring system for three lengths $(6,9$ and 12 times the diameter of the rod) with two rod diameters (3/8" y 5/8") in two types of high-strength concrete (5000Psi y 6000Psi) to determine the length and effective system load. The analysis carried out on the experimental test is to correlate the result of the maximum strength of steel reinforcement used (fy $=4200 \mathrm{Psi}$ ) with the variables post anchor system installed. The results obtained are the percentage of system resistance depending on anchor length; the length that ensures steel fluency and their correlations between the percentage of effective resistance reached for each anchorage length and the use of high strength concrete. With the above parameters, the contribution of high strength concretes to the efficiency of the installed anchorage systems was analyzed.

Keywords: Post-installed anchor, high strength concrete.

\section{INTRODUCCIÓN}

Con todo el auge de la construcción, no hay que perder de vista que hoy en día las exigencias de las estructuras de infraestructura vial están haciendo que cada vez sea más frecuente el uso de concretos de alta resistencia. Los puentes con altas exigencias mecánicas, los pavimentos con edades tempranas de servicio, las estructuras con geometrías cada vez más esbeltas, los elementos preesforzados o postensados en estructuras de infraestructura vial, los puertos con estructuras sujetas a condiciones severas de exposición, como ambientes agresivos marinos, son algunas de las obras de infraestructura vial que han fomentado el uso de concretos con condiciones de alta resistencia; sin embargo, el estudio del uso de an- 
clajes epóxicos posinstalados en concretos con estas exigencias no se ha profundizado.

En todos los sectores de la construcción de infraestructura vial se realiza la reutilización de obras actuales o continuación de las construcciones de concreto existente, se generan juntas de construcción, se efectúan ampliaciones, se hacen rehabilitaciones, se construyen reforzamientos, se desarrollan rediseños o por el proceso constructivo se hacen construcciones segmentadas, como es el caso de los puentes. Es por esto que se hace innegable el uso de anclajes posinstalados entre las estructuras existentes y las nuevas que garanticen que se comporten adecuadamente (adherencia, transferencia de cargas o garantizar estructuras monolíticas). De estos anclajes el más utilizado es el anclaje epóxico.

Por lo anterior, el objetivo de la investigación es evaluar la longitud y resistencia efectivas de los anclajes epóxicos posinstalados para dos diámetros de varilla corrugada, en probetas de dos calidades de concreto de alta resistencia mediante ensayos a tracción directa.

\section{INVESTIGACIONES PRECEDENTES}

En los últimos años, se han realizado numerosos estudios para investigar el comportamiento de los anclajes adhesivos (anclajes posinstalados), en especial, los efectos de la longitud de empotramiento (longitud de anclaje), el tipo de agente de anclaje utilizado (adherente), diámetro de la perforación, diámetro de la barra de refuerzo, distancia al borde y separación de los anclajes en una superficie de concreto.
Hay pruebas de extracción total realziadas por Shah et al. [1] sobre barras de acero anclado en dos longitudes de empotramiento diferentes (longitud de anclaje), utilizando materiales de dos fabricantes diferentes. Obata et al. [2] han desarrollado estudios de los efectos de borde libre en la resistencia a la tracción de los sistemas de anclaje, tanto experimental como analíticamente [3].

También se ha investigado el comportamiento de los anclajes posinstaladas de piedra natural [4] y mampostería [5]; al mismo tiempo, algunos estudios han investigado el comportamiento de los anclajes adhesivos (anclajes posinstalados) bajo carga externa. El comportamiento a la tracción de anclajes epóxicos posinstalados incrustados en el concreto de baja resistencia también se ha estudiado [6], al igual que el comportamiento de cizallamiento de los anclajes epóxicos embebidos en el concreto de baja resistencia [7]. Kwon et al. [8] han estudiado el comportamiento de conectores de corte postinstaladas bajo cargas estáticas y de fatiga. También han sido reportados los modos de falla, con una descripción completa de los efectos de la tasa de carga en función del comportamiento de soporte de los anclajes [9]. Varios modos de fallo [9][10] se han propuesto en la literatura para determinar la falla a la carga de los anclajes adhesivos (anclajes post instalados) en el concreto. En la mayoría de los casos, estos modos están relacionados con productos específicos y el diámetro de las barras de ensayo por utilizar es normalmente de menos de $40 \mathrm{~mm}$. Se investigó la fuerza de extracción para los anclajes posinstalados de gran diámetro incrustados en cimientos de concreto [11]. También se encontró que 
el $\mathrm{ACl} 318$ tiene un enfoque muy sobreconservador para la capacidad de tracción de los anclajes posinstalados cuando se considera un concreto de baja resistencia; además, ha congregado la mayor cantidad de información para las investigaciones de los sistemas de anclajes posinstalados a través del informe del estado del arte de los anclajes en hormigón (concreto) [12].

Las investigaciones desarrolladas, aunque no han usado concreto de alta resistencia, han logrado caracterizar algunas de las propiedades mecánicas, clasificar tipos de anclajes, desarrollar metodologías de ensayo, y hasta recomendar procesos de instalación [13]. Como síntesis de todo lo anterior, se desarrolla la consulta e integración del estado del arte, de las variables que intervienen en un sistema de anclaje posinstalado, con el objeto de parametrizar las variables por tener en cuenta en el presente estudio, sin detenerse en estudios de simulación numérica y comportamiento a corte o químico del sistema de anclajes posinstalados y otros no mencionados.

\section{PROCEDIMIENTO EXPERIMENTAL}

\subsection{Fabricación del concreto de alta resistencia}

Para el proyecto se utilizaron dos resistencias de concreto definidas como $f^{\prime}{ }_{c 1} y^{\prime}{ }^{\prime}$. Se fabricaron 60 probetas, 30 de cada resistencia correspondientes al tamaño de la muestra, y 6 probetas adicionales para imprevistos. Se tomaron las recomendaciones para la construcción de concretos de alta resistencia, de acuerdo con los catálogos encontrados acerca de los concretos especiales en la construcción de concretos de alta resistencia [14]-[15]. De acuerdo con lo anterior, se decidió usar cemento portland convencional, verificar las dosificaciones para alcanzar concretos de resistencia alta y definir una relación agua-cemento $A / C$ que garantice un concreto con resistencia alta [16]. Por esto, se encontró que para obtener resistencias superiores a $34 \mathrm{MPa}$, se requieren relaciones de agua-cemento inferiores a 0,45 [17]. En la práctica estas relaciones A/C son un poco complicadas de obtener; por tanto, fue necesario el uso de un reductor de agua para lograr las relaciones especificadas [18].

Por otro lado, se midió la resistencia a la compresión a los 7 días para verificar la proyección de resistencia esperada a los 28 días, y luego del periodo de curado se midió a los 28 días la resistencia a la compresión de 6 probetas ( 3 probetas de cada resistencia), en la máquina universal de la Universidad Pedagógica y Tecnológica de Colombia, empleando la norma NTC 673, ensayo de resistencia a la compresión de cilindros de concreto, con lo cual se verificó que las resistencias del concreto son las esperadas para el desarrollo de la investigación [19].

Teniendo en cuenta la proyección de los resultados de los ensayos a 7 días, se logró predecir la resistencia a los 28 días de cada una de las resistencias de trabajo. Como resultado, la resistencia $\mathrm{f}_{\mathrm{c} 1}^{\prime}$ fue de $34,38 \mathrm{MPa}$ (5000Psi) y la resistencia $\mathrm{f}_{\mathrm{c} 2}$, de $42,11 \mathrm{MPa}$ (6000Psi) 
2.2. Proceso de ensamble de anclajes posinstalados

El procedimiento de ensamble de los sistemas de anclaje posinstalado fue tomado y adaptado del proyecto de investigación que se titula Estudio de la resistencia a tracción en anclajes estructurales post instalados con adhesivo epóxico [20], de la línea de investigación en la cual se enmarcó el presente proyecto; además, se tomaron en cuenta los factores que influyen en el sistema [21], así como las recomendaciones de los elementos que afectan la resistencia a traccion de esta [22]. Se realizaron los sistemas de anclaje de acuerdo con el siguiente diseño experimental.

Se usaron barras de refuerzo de $3 / 8^{\prime \prime}$ $(9,5 \mathrm{~mm})$ y $5 / 8^{\prime \prime}(15,9 \mathrm{~mm})$. El diámetro de la perforación se realizó usando brocas de diámetros $1 / 2$ " para anclaje de $3 / 8^{\prime \prime}$ y $3 / 4$ " para anclaje de 5/8"; es decir, 1/8" adicional al diámetro de la barra de refuerzo. Las longitudes de perforación usadas fueron 6,9 y 12 veces el diámetro de la varilla. Por tanto, las longitudes de perforación fueron para la barra de refuerzo de $3 / 8^{\prime \prime}(9,5 \mathrm{~mm})$ : 6 veces $=57 \mathrm{~mm}$; 9 veces $85 \mathrm{~mm}$ y 12 veces $114 \mathrm{~mm}$, y para la barra de refuerzo de 5/8" (15,9mm): 6 veces $=95,4 \mathrm{~mm}$; 9 veces $143 \mathrm{~mm}$ y 12 veces 190,8mm. Los sistemas de anclaje se instalaron en los concretos $f_{c 1}^{\prime}(5000 \mathrm{Psi}) \mathrm{y}$ $f_{c 2}^{\prime}(6000 P s i)$. Lo anterior corresponde a un diseño experimental factorial $3 \times 2 \times 2$ con 4 repeticiones, es decir, 3 longitudes de perforación, 2 diámetros de varilla, en 2 calidades de concreto con 4 repeticiones de cada combinación, para un total de 48 probetas. El montaje de los sistemas de anclajes con las principales variables para el ensamble se realizó como se muestra en la Fig. 1.

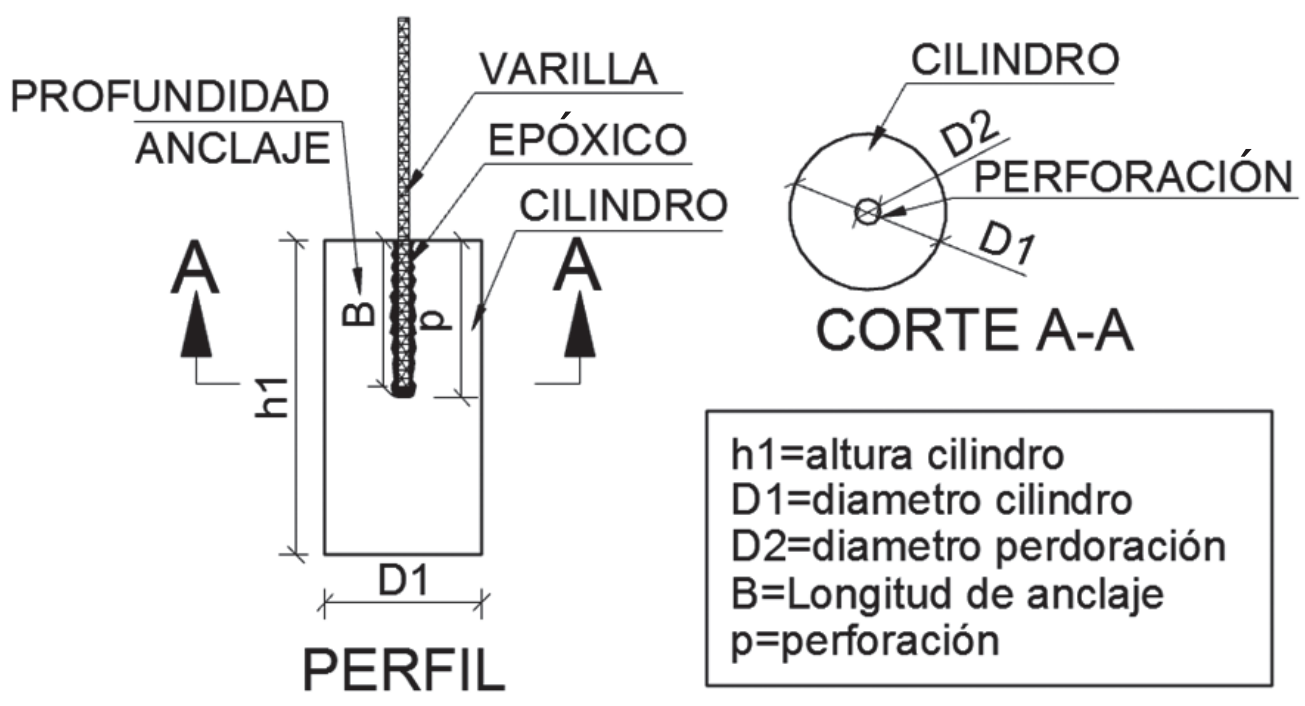

Fig. 1. Sistema de anclaje postinstalado

Fuente: elaboracipon propia. 
Posteriormente, se procedió a la instalación de las barras de refuerzo con adhesivo epóxico (como se muestra en la Fig. 1), para lo cual se tomaron en cuenta las recomendaciones del fabricante [23], para garantizar los requisitos de funcionamiento de los adhesivos estructurales [24].

Luego de ensambladas las probetas de sistemas de anclaje, se efectuaron las pruebas de resistencia a tracción. Los ensayos de resistencia a tracción pura en los sistemas de anclaje estructural se realizaron en la máquina universal del laboratorio de suelos y materiales de la Universidad Pedagógica y Tecnológica de Colombia (UPTC).
Para el montaje se fabricó un acople de acero ajustado a la máquina universal del laboratorio, el cual dispone un orificio circular dispuesto en el centro geométrico de $0,12 \mathrm{~m}$ de diámetro para generar un área libre para el desarrollo de la falla del sistema. Se hizo un ensayo típico a tracción, a una velocidad de carga de $1 \mathrm{MPa} / \mathrm{s}$, y se fallaron las 48 probetas. Con esto se obtuvo como resultado para cada espécimen la gráfica carga $(\mathrm{KN})$ versus alargamiento $(\mathrm{mm})$, característica de esta prueba. Este procedimiento se ejecutó con base en Icontec (1995). El esquema de montaje se presenta en la Fig. 2.

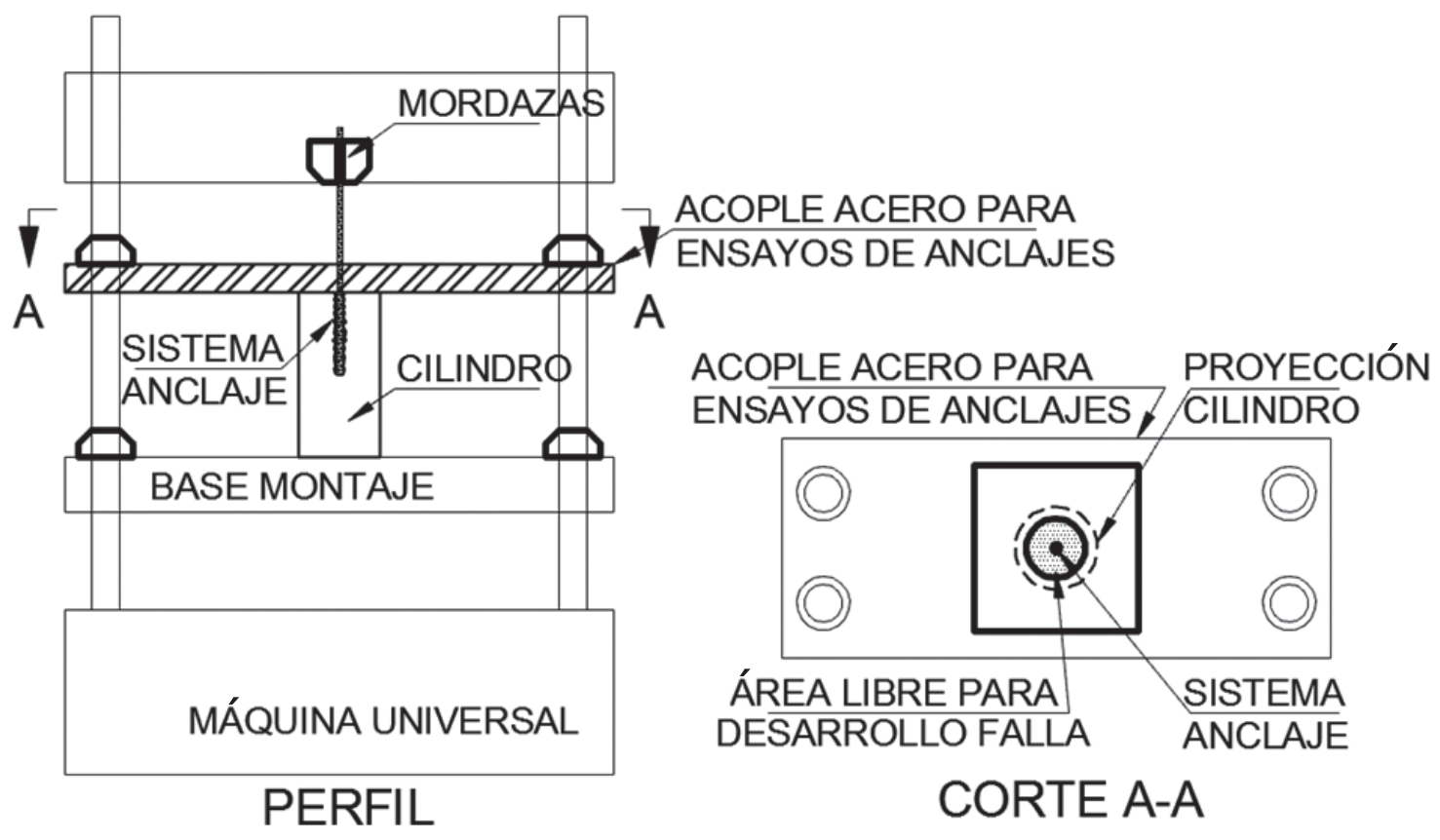

Fig. 2. Esquema de montaje ensayo a tracción de sistema anclaje posinstalado.

Fuente: elaboración propia. 


\section{EVALUACIÓN DE LA LONGITUD EFECTIVA}

Para las dos resistencias de concreto se fallaron las probetas con las tres profundidades para evaluar la longitud efectiva de anclaje con barras de refuerzo de $3 / 8$ " $(9,5 \mathrm{~mm})$ y $5 / 8^{\prime \prime}(15,9 \mathrm{~mm})$. Como base para correla- cionar los resultados de los sistemas de anclaje posinstalado, se realizaron las pruebas a tracción del acero de refuerzo utilizado [25]. En la Fig. 3 se muestran los resultados de la curva de resistencia a tracción del refuerzo $3 / 8^{\prime \prime}(9,5 \mathrm{~mm})$ a la izquierda y a la derecha, la curva de resistencia a tracción $5 / 8^{\prime \prime}$ $(15,9 \mathrm{~mm})$.

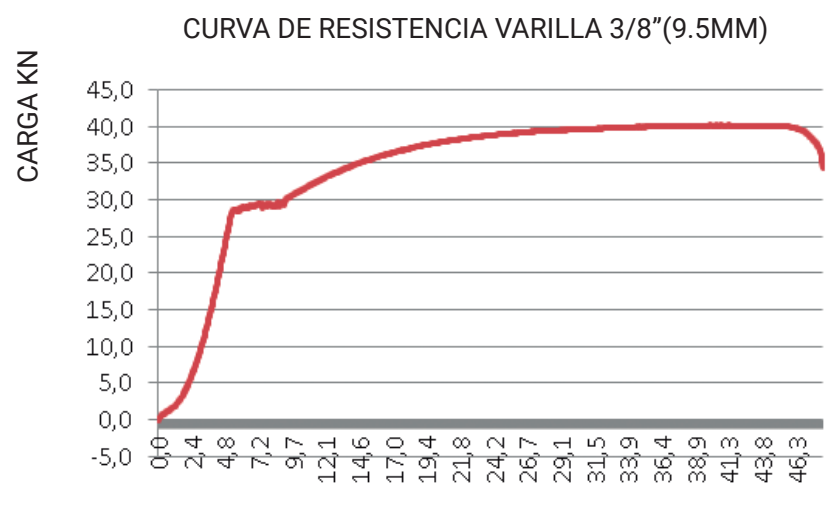

ALARGAMIENTO (mm)

CURVA DE RESISTENCIA VARILLA 5/8"

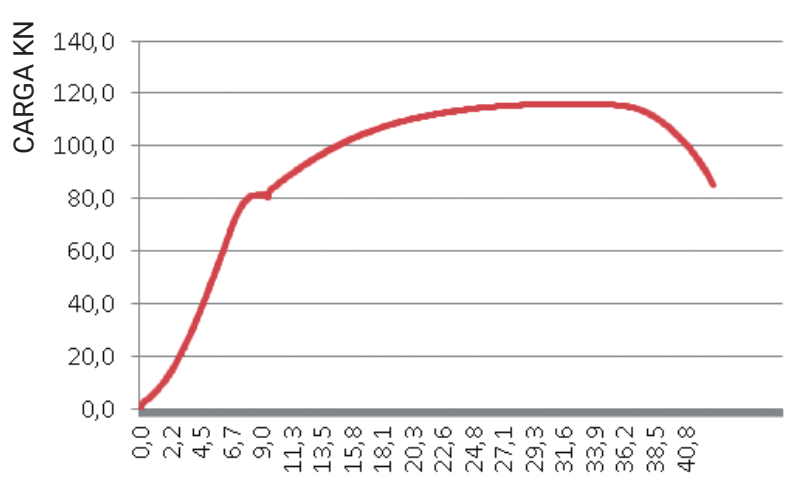

ALARGAMIENTO

Fig. 3. Curvas de resistencia tracción 3/8" (9,5mm) y 5/8 (15,9mm)

Fuente: elaboracipon propia. 
De las curvas de resistencia de las barras acero, se identificó el parámetro "base" de la correlación de longitud efectiva de anclaje. De las muestras de control que aparecen en la Fig. 3, se definió como principal parámetro la carga máxima de fluencia del acero. Tomando como base el valor mencionado, se buscó que el sistema de anclaje posinstalado garantizara la condición con la cual la barra de refuerzo del sistema de anclaje sea capaz de desarrollar la carga máxima de influencia del refuerzo correspondiente a la probeta ensamblada. Los valores de resistencia de "carga máxima de fluencia" correspondientes a cada varilla fueron $3 / 8^{\prime \prime}(9,5 \mathrm{~mm})=29,43$ KN y 5/8" $(15,9 \mathrm{~mm})=81,73 \mathrm{KN}$. Se analizó la longitud de anclaje (perforación) en función del porcentaje de resistencia alcanzado para cada resistencia de concreto, con cada varilla de refuerzo según la profundidad de la perforación (veces el $\varnothing$ de la varilla), como se muestra en las Figs. 4, 5 y 6 . El porcentaje desarrollado del sistema de anclaje respecto a la carga de fluencia del acero se calcula con el promedio aritmético de las probetas según su diámetro y profundidad de anclaje.
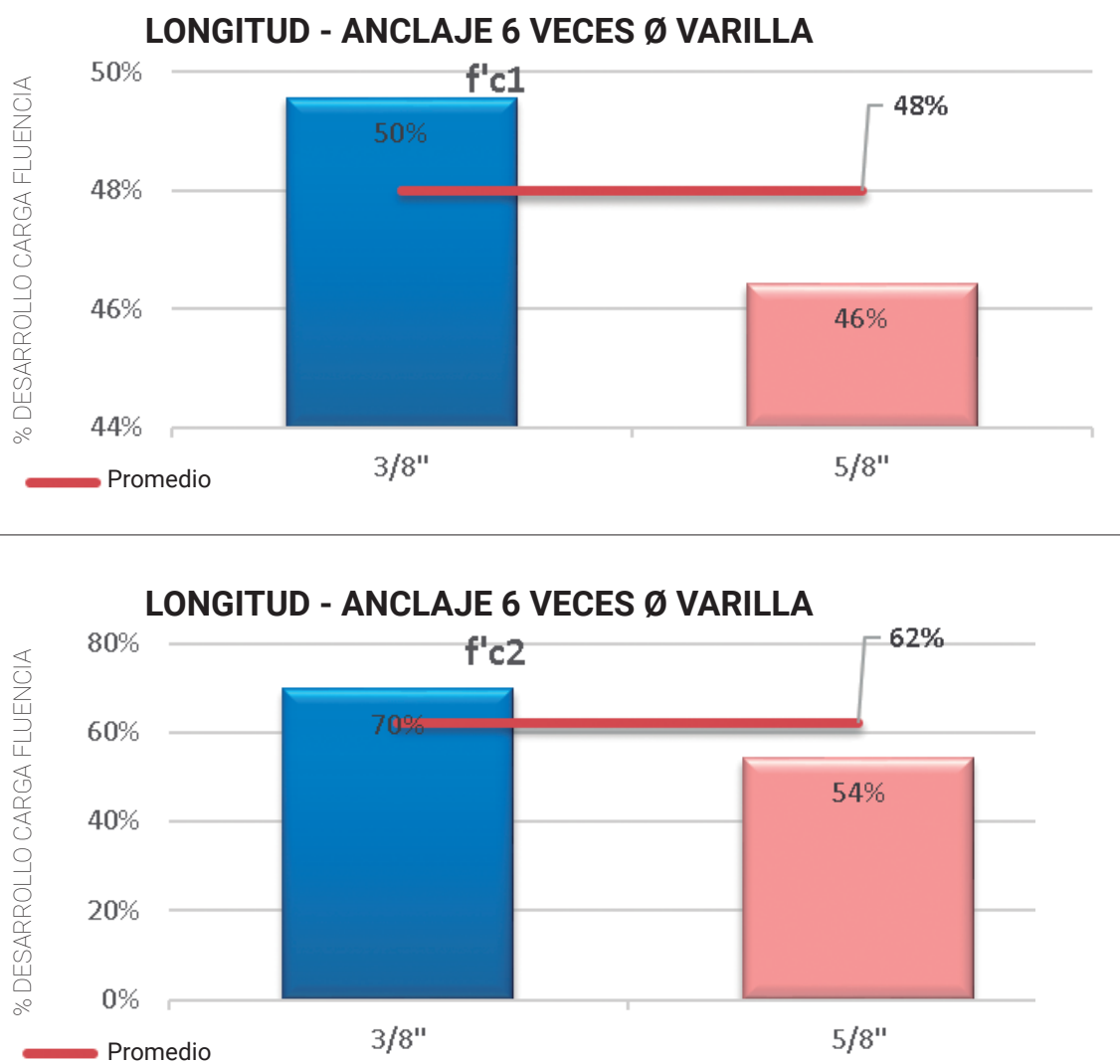

Fig. 4. Longitud de anclaje seis veces el diámetro, promedio resistencia Fuente: elaboracipon propia. 
Se estableció que para la resistencia de concreto $\mathrm{f}^{\prime}{ }_{\mathrm{c} 1}$, tomando una longitud de anclaje de 6 veces el diámetro de la varilla, se desarrolla en promedio el $48 \%$ de la carga máxima a fluencia del acero de refuerzo. Para una lon- gitud de anclaje de 9 veces el diámetro de la varilla se desarrolla en promedio el $73 \%$; finalmente, para la longitud de anclaje de 12 veces el diámetro de la varilla se desarrolla en promedio el $92 \%$.
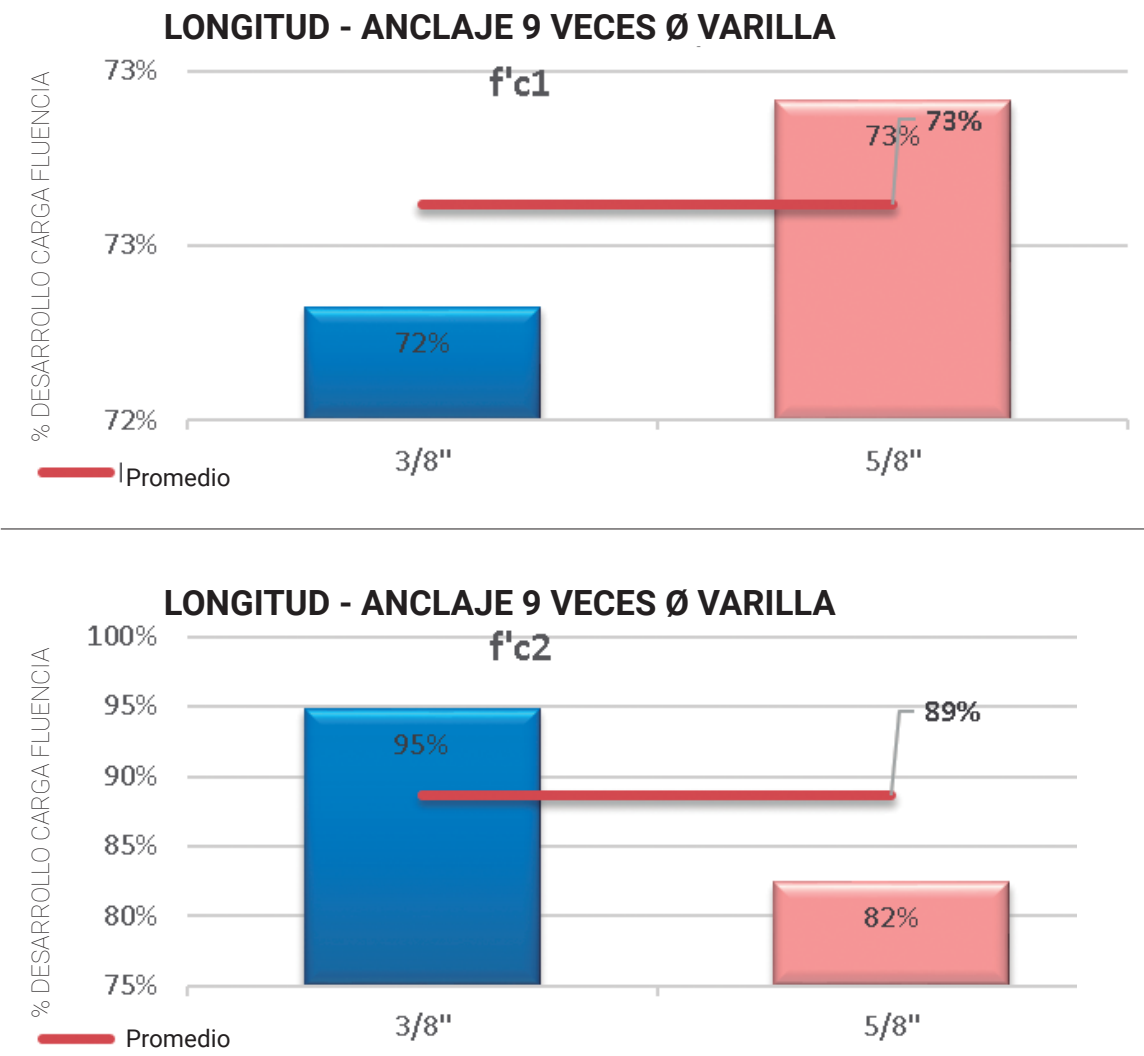

Fig. 5. Longitud de anclaje nueve veces el diámetro, promedio resistencia Fuente: elaboracipon propia. 

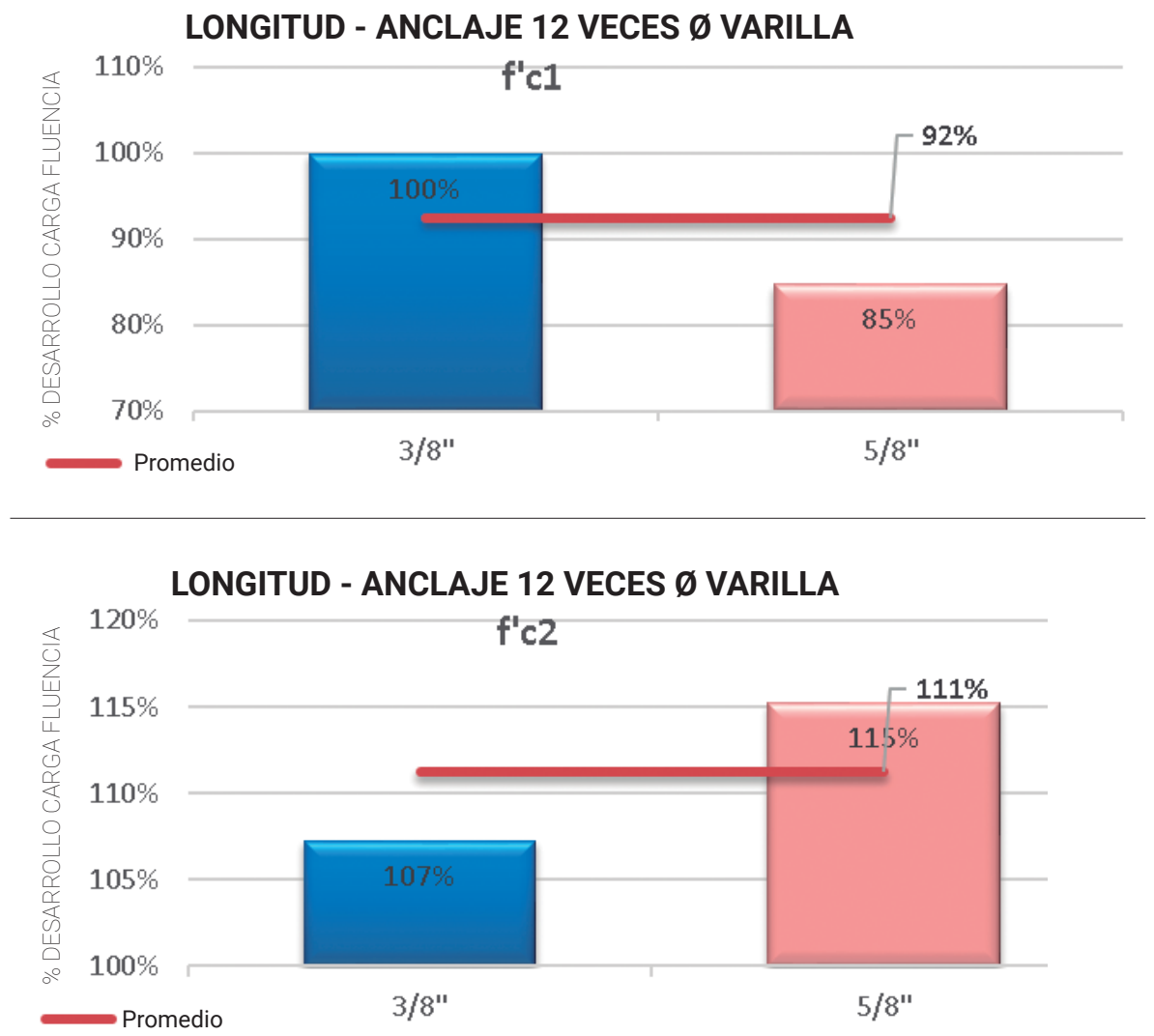

Fig. 6. Longitud de anclaje doce veces el diámetro, promedio resistencia Fuente: elaboracipon propia.

Por lo anterior, se determina que con una longitud de anclaje de 12 veces el diámetro de la varilla se cumple de forma "aceptable" con la resistencia máxima de fluencia del acero de refuerzo. Para la resistencia de concreto $f^{\prime}{ }^{\prime}$, tomando una longitud de anclaje de 6 veces el diámetro de la varilla, se desarrolla en promedio el $62 \%$ de la carga máxima a fluencia del acero de refuerzo. Para una longitud de anclaje de 9 veces el diámetro de la varilla se desarrolla en promedio el $89 \%$ de la carga máxima a fluencia del acero de refuerzo; finalmente, para la longitud de an- claje de 12 veces el diámetro de la varilla, se desarrolla en promedio el $111 \%$ de la carga máxima a fluencia del acero de refuerzo. En vista de lo mencionado, se comprueba que con una longitud de anclaje de 12 veces el diámetro de la varilla, se cumple ampliamente con la resistencia máxima de fluencia del acero de refuerzo.

El porcentaje de la carga máxima del sistema de anclaje depende del diámetro de la varilla; es decir, que a menor diámetro se va a desarrollar una mayor resistencia del sistema de anclaje. Por esto, una 
vez definida el área de refuerzo, será más conveniente usar una mayor cantidad de acero de menor diámetro, como se mencionó en el artículo del estudio "Behavior of post-installed large-diameter anchors in concrete foundations" [11], donde se indicó que al aumentar el diametro de refuerzo, aumentaba marginalmente la resisetencia del sistema de anclaje. Otra correlación que se analizó fue la longitud de anclaje en función de la resistencia del concreto, la cual se muestra en la Fig. 7. Asimismo, se realizó el promedio de los resultados de anclaje para las probetas con seis veces el diámetro de la varilla. El proceso anterior se realizó también para los resultados de anclaje para las probetas con nueve y doce veces el diámetro de la varilla. Este indicador se calculó para cada una de las resistencias, de tal manera que los resultados pudieran ser comparables. Se obtuvo con esto lo que se muestra en la Fig. 7.

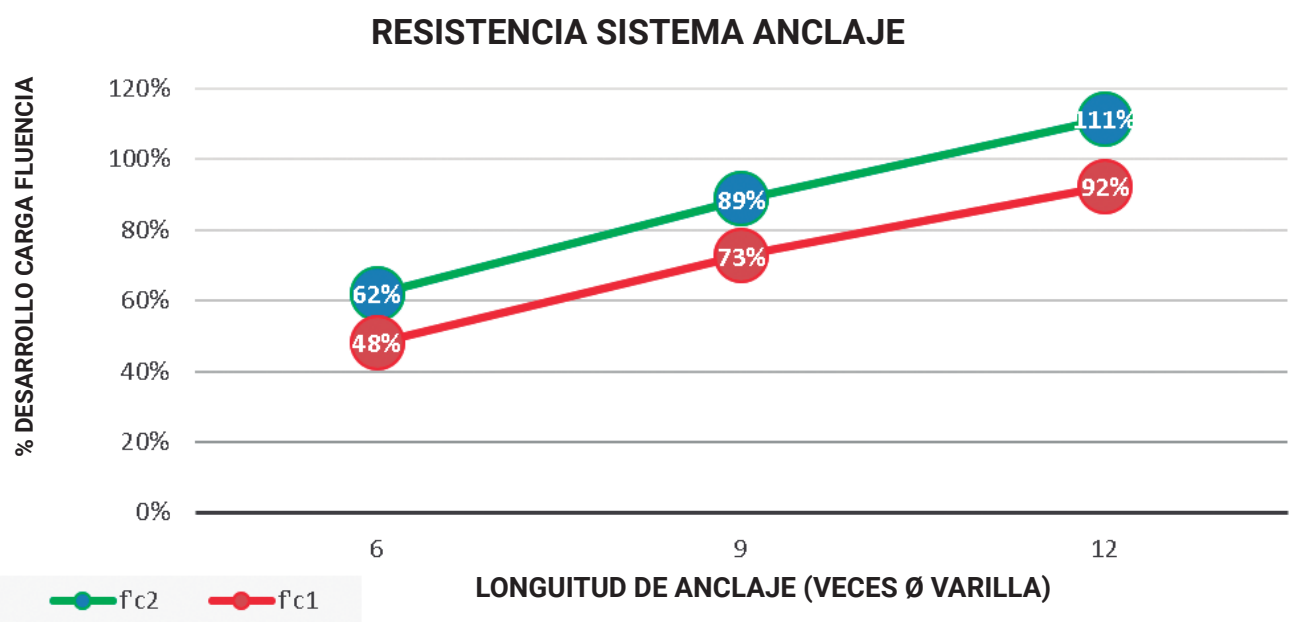

Fig. 7. Resistencia según longitud de anclaje para cada f'

Fuente: elaboracipon propia.

La longitud de anclaje tiene un mejor comportamiento dependiendo de la resistencia del concreto. Como se observa en la Fig. 7 , el sistema de anclaje con 6, 9 y 12 veces la longitud de anclaje de la varilla para la resistencia $f_{c 2}^{\prime}$ es mayor en todos los casos a las resistencias desarrolladas para $\mathrm{f}_{\mathrm{c} 1}^{\prime}$. De lo anterior se podría indicar de forma preliminar que por cada 1000Psi de resistencia en los concretos de alta resistencia, se podría mejorar el desarrollo de resistencia a la carga en un $15 \%$. Lo anterior coincide con los estudios que determinan que en diferentes calidades de materiales, cambia la resistencia del sistema de anclaje [1]. Por otra parte, la tendencia de la longitud de anclaje en función de $n$ (veces el diámetro de la variIla) es lineal, por lo que a través de ensayos 
como los propuestos en el presente estudio experimental y realizando la metodología de análisis propuesta, se podría determinar la longitud efectiva para cualquier sistema de anclaje posinstalado con una resistencia de concreto $\mathrm{f}_{\mathrm{c}}$ definida.

Al correlacionar el comportamiento obtenido en el ensayo con los estudios similares desarrollados, se pudo validar que en concretos de alta resistencia aplica el diseño de los anclajes adhesivos individuales en concreto no fisurado [26].

\section{EVALUACIÓN DE LA RESISTENCIA EFECTIVA}

Se graficaron las curvas de resistencia de los resultados obtenidos en los ensayos para 12 veces el diámetro de la varilla con un acero de refuerzo $3 / 8$ " $(9,5 \mathrm{~mm})$ (Fig. 8) y $5 / 8$ " (15,9mm) (Fig. 9), para observar el comportamiento durante el ensayo de tracción de los sistemas de anclajes [27]. Para cada grupo de resistencia de concreto $f^{\prime}{ }_{c 1} y f^{\prime}{ }^{\prime 2}$, se identifica cada una de las probetas para correlacionarlas entre ellas.

CURVA DE RESISTENCIA f'c1
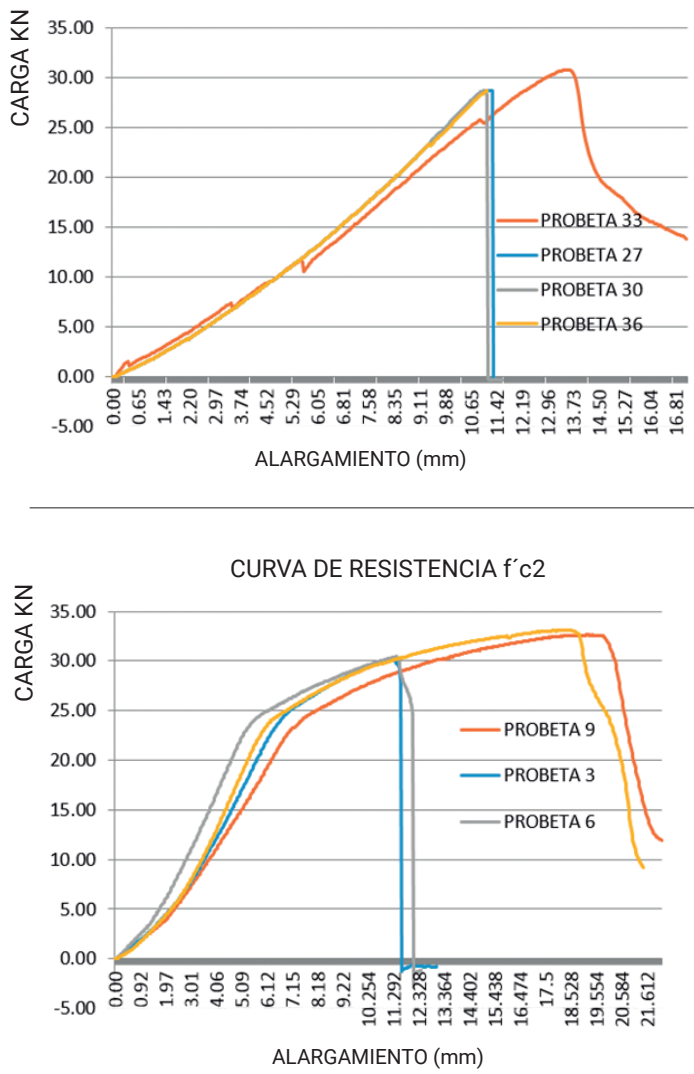

Fig. 8. Curvas de resistencia $f_{c 1}^{\prime}(3 / 8 " 9,5 \mathrm{~mm})$ y $f_{c 2}^{\prime}(3 / 8 " 9,5 \mathrm{~mm})$

Fuente: elaboracipon propia. 

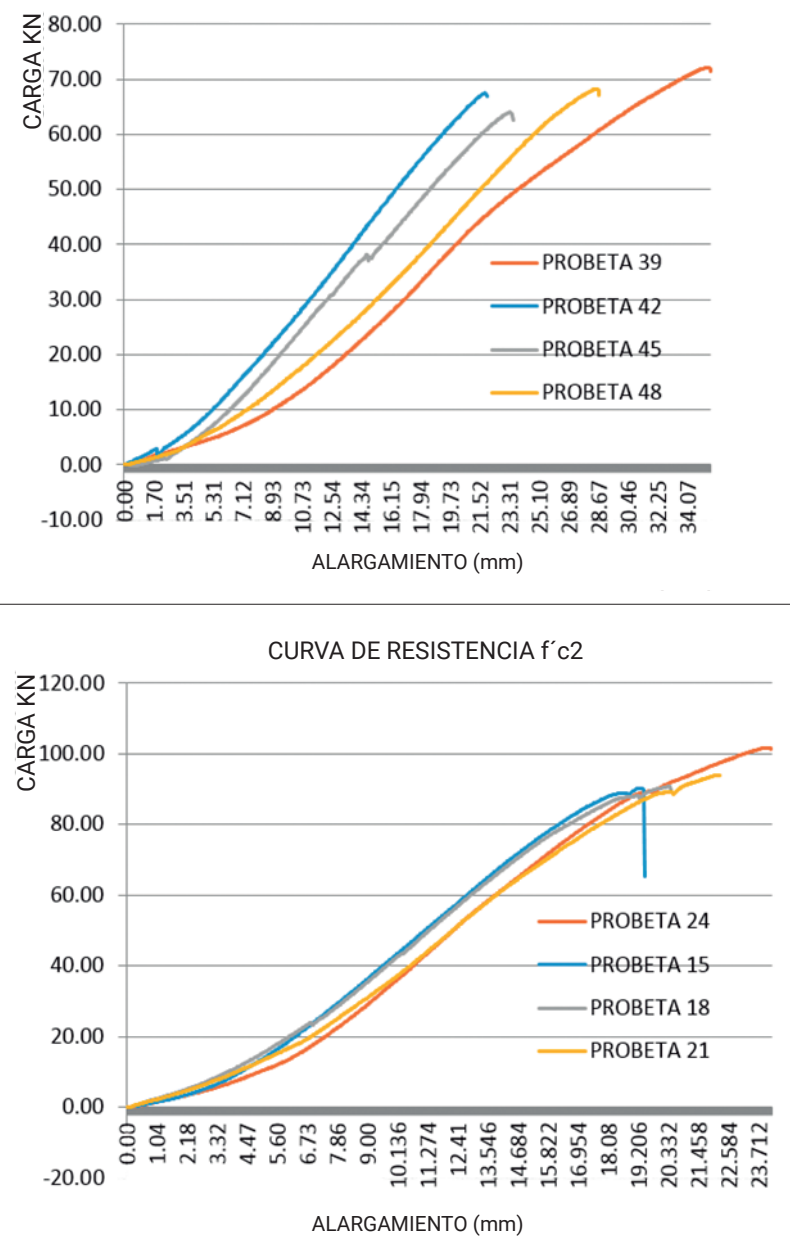

Fig. 9. Curvas de resistencia $\mathrm{f}_{\mathrm{c} 1}^{\prime}\left(5 / 8^{\prime \prime} 15,9 \mathrm{~mm}\right)$ y $\mathrm{f}_{\mathrm{c} 2}\left(5 / 8{ }^{\prime \prime} 15,9 \mathrm{~mm}\right)$

Fuente: elaboracipon propia.

En el comportamiento para las barras $3 / 8^{\prime \prime}$ $(9,5 \mathrm{~mm})$ de diámetro (Fig. 8), se pudo identificar la semejanza al comportamiento de curva de resistencia del acero [28], mientras que para las barras 5/8" (15,9mm) de diámetro (Fig. 9), se desarrollaron las gráficas en la zona elástica, hasta la falla del concreto en el sistema de anclaje. En general, basados en las curvas mostradas se puede identificar la zona elástica, la carga máxima de fluencia, la zona plástica y la carga de rotura del acero de refuerzo. El punto donde la gráfica decrece indica el instate de falla de la probeta o punto de rotura de la barra de refuerzo. Correlacionando lo anterior con un estudio hecho en concretos de baja resistencia, usar 
barras de menor diámetro podría proporcionar diseños más seguros, ya que los menores diámetros de acero son más suseptibles a desarrollar por completo la curva de resistencia hasta la carga de rotura [7].

Al realizar la comparación entre el grupo de resultados de las cargas a tracción de la resistencia de concreto $f^{\prime}{ }_{\mathrm{c} 2}$ y $f^{\prime}{ }_{\mathrm{c} 1}$, se observa que en las curvas del concreto de mayor resistencia se desarrollan unas mayores cargas máximas del sistema de anclaje. Aun cuando la falla sea por el concreto o por el acero en el sistema de anclaje, en los dos casos el acero de refuerzo ya había alcanzado la resistencia máxima de fluencia $\mathrm{fy}=4200 \mathrm{k} /$ $\mathrm{cm}^{2}$. También se puede afirmar que en cualquiera de los dos casos se garantizaría que el sistema de anclaje siempre cumpla con los requisitos de resistencia.

Tanto en la Fig. 8 como en la Fig. 9, se identificó que para los sistemas de anclaje posinstalado, en su mayoría, las probetas fallaron por el concreto, mientras que en la curva de resistencia se identifica como el cambio de pendiente subito (decreciente), o tambien se puede reconocer cuando el punto de culminacion de la curva de resistencia ha terminado y no mostró el desarrollo de la curva de resistencia completa del acero de refuerzo, mostrado en la Fig. 3 (muestras de control).

Al haber predefinido en el presente proyecto de investigación tomar 12 veces el diámetro para establecer la resistencia efectiva del sistema de anclaje (el cual desarrolle el $100 \%$ de la carga máxima de fluencia) y observando los resultados de los ensayos a tracción donde su gran mayoría fallaron por el concreto (aunque se está garantizando que el sistema de anclaje funciona), la falla podría no ser dúctil. Lo anterior se menciona teniendo en cuenta el escenario de la metodología experimental propuesta en el proyecto, es decir, usando probetas de concreto simple con un sistema de anclaje. Además, es importante tener en cuenta que la fabricación de las probetas no considera variables como el confinamiento del concreto con acero de refuerzo, como se estudió en Contrastación experimental de la resistencia de anclajes epoxicos [29], donde sí se consideraron las probetas con refuerzo de confinamiento, como en los elementos de un sistema de concreto reforzado.

Por otro lado, la resistencia efectiva a tracción en anclajes posinstalados, a través de ensayos de tracción directa, dió como resultado que depende directamente de la longitud de anclaje. Finalmente se realiza el cálculo de la resistencia efectiva a tracción en función de la profundidad de anclaje para cada barra de refuerzo. El cálculo se hace con el promedio aritmético de los resultados de las cargas máximas registradas en los ensayos en las dos calidades de concreto $f^{\prime}{ }_{c 1}$ $y f_{c 2,}^{\prime}$ y se muestran en las Figs. 10, 11 y 12 . 


\section{Ciencia e Ingeniería Neogranadina}
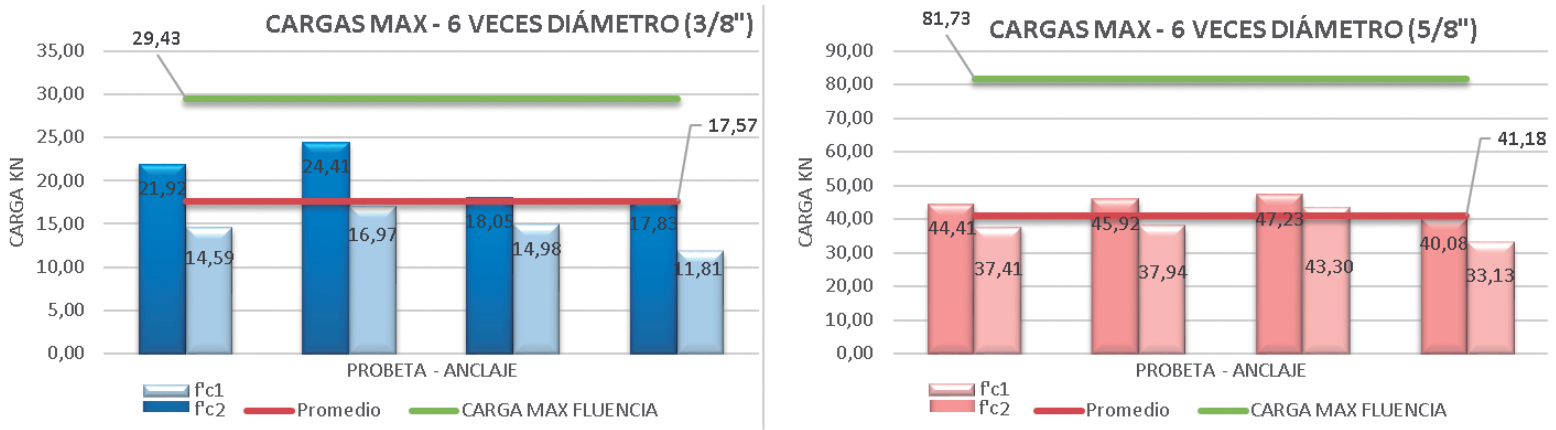

Fig. 10. Resistencia efectiva (profundidad de anclaje: seis veces el diámetro varilla) Fuente: elaboracipon propia.
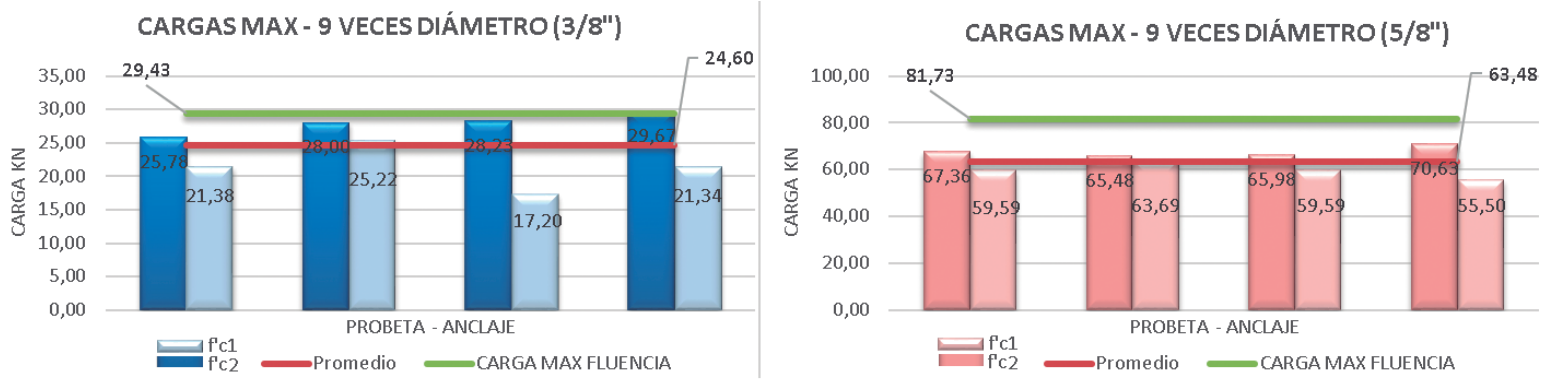

Fig. 11. Resistencia efectiva (profundidad de anclaje: nueve veces el diámetro varilla)

Fuente: elaboracipon propia.
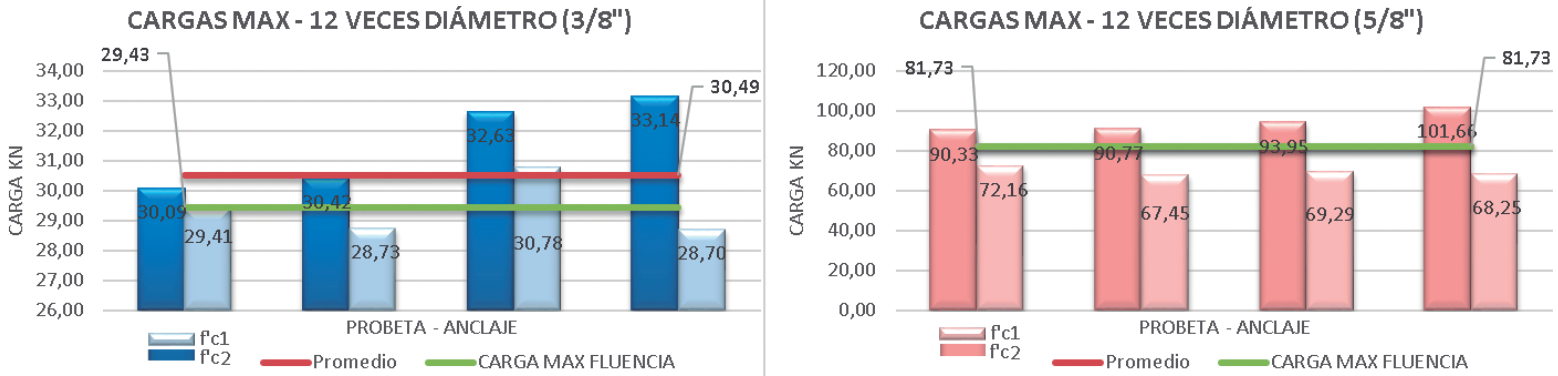

Fig. 12. Resistencia efectiva (profundidad de anclaje: doce veces el diámetro varilla) Fuente: elaboracipon propia. 
Como resultado del análisis, se determinó que con una longitud de anclaje de seis veces el diámetro de la varilla la resistencia efectiva a tracción desarrolla entre un $50 \%$ y un $60 \%$ de la carga máxima de fluencia del acero de refuerzo (ver Fig. 10). Con una longitud de anclaje de nueve veces el diámetro de la varilla, la resistencia efectiva a tracción desarrolla alrededor del $80 \%$ de la carga máxima de fluencia del acero de refuerzo (ver Fig. 11). Finalmente, con una longitud de anclaje de doce veces el diámetro de la varilla, la resistencia efectiva a tracción garantiza el $100 \%$ de la carga máxima de fluencia del acero de refuerzo (ver Fig. 12). Para el caso de estudio, siendo la carga máxima del acero de refuerzo entre $30 \%$ y $40 \%$ mayor a la carga máxima de fluencia, el sistema de anclaje estaría supeditado a no superar estos valores.

\section{CONCLUSIONES}

En las investigaciones precedentes, se observó que los estudios de los anclajes posinstalados han crecido, en función de su uso en las obras de construcción, y la longitud de anclaje es la variable más analizada [1], y, como se comprobó en la presente investigación, resulta ser la más sensible en los análisis de resultados.

Teniendo en cuenta que en la actualidad la producción de los concretos de alta resistencia se están dirigiendo a proyectos de infraestructura vial y que además el uso de anclajes posinstalados es más frecuente durante el proceso constructivo, se justificó en esta investigación la evaluación de los anclajes posinstalados en concretos de alta resistencia.
En concretos de alta resistencia, los sistemas con longitud de anclaje de nueve veces el diámetro de la varilla, con refuerzo de diámetro igual o menor a $3 / 8$ " podrían satisfacer los requerimientos de diseño, pues se encontró que con esta profundidad se puede llegar a desarrollar un mínimo del $85 \%$ de la resistencia del acero de refuerzo. Lo anterior se menciona en caso de que se aplicase de forma similar la condición establecida en la NSR10- Paragrafo C5.6.5.4, donde se aprueba una muestra con el $85 \%$ de la resistencia (del concreto) [30].

De los resultados de las cargas máximas de los sistemas de anclaje, se observó que en los sistemas de anclaje con doce veces el diámetro de la varilla se garantiza que se desarrolle la carga máxima de fluencia del acero de refuerzo.

Al analizar la longitud de anclaje con el diámetro de la varilla, pudo verse que a menor diámetro de la barra, el sistema de anclaje posinstalado desarrolla mayor resistencia a tracción. Lo anterior ratifica que después de definida un área de refuerzo será más conveniente usar una mayor cantidad de acero de menor diámetro [7].

Se identificó que la longitud efectiva de anclaje para dos diámetros de varilla corrugada, en dos concretos de resistencia alta mediante ensayos a tracción directa, es once veces el diámetro de la varilla. Las recomendaciones del proveedor [23], al definir la longitud de anclaje como doce veces el diámetro de la varilla, garantizarían que el acero de refuerzo satisfaga las solicitaciones de resistencia. Para el caso de estudio, al aumentar la calidad del concreto, aumenta la resistencia a tracción de los sistemas 
de anclajes posinstalados. Se observó que por cada 1000Psi de resistencia en los concretos de alta resistencia, se podría mejorar la capacidad de resistencia a la carga de los anclajes posinstalados en un $15 \%$.

Aun cuando la falla en las probetas del sistema de anclaje sea por el concreto o por el acero, con una profundidad de anclaje de once veces el diámetro del acero de refuerzo, alcanzará la resistencia máxima de fluencia $f y=4200 \mathrm{k} / \mathrm{cm}^{2}$. Es por esto que se puede definir que en cualquiera de los dos casos se garantizaría que el sistema de anclaje siempre cumpla con los requisitos (solicitaciones) de resistencia. Se aclara que las pruebas se desarrollaron con probetas de concreto simple y no concreto confinado, como se ha considerado en algunos estudios donde se integra de forma implícita dicha variable [29].

Adicionalmente, se identificó que con una longitud de anclaje de seis veces el diámetro de la varilla, la resistencia efectiva a tracción desarrolla entre un $50 \%$ y un $60 \%$ de la carga máxima de fluencia del acero de refuerzo. Con una longitud de anclaje de nueve veces el diámetro de la varilla, la resistencia efectiva a tracción desarrolla alrededor del $80 \%$. Finalmente, se determinó que con una longitud de anclaje de 12 veces el diámetro de la varilla, la resistencia efectiva a tracción garantiza el $100 \%$ de la carga máxima de fluencia del acero de refuerzo.

Frente a una mayor calidad del concreto, aumenta la resistencia del sistema de anclaje posinstalado, como se mostró en el estudio al comparar los sistemas de anclaje en los concretos de alta resistencia usados en el caso de estudio. Lo anterior se menciona al correlacionar los resultados de estracción de anclajes en diferentes calidades de materiales, en los estudios realizados en concreto de baja resistencia, concreto estandar, piedra [4] y mampostería [5].

\section{AGRADECIMIENTOS}

A Dios que no permite que nos rindamos (Fil. 4-13). A mi familia, al equipo de trabajo, desde el director, el ingeniero Diego Paez Moreno, hasta la última persona que se me pueda escapar en este breve mensaje. A la institución que represento, la Universidad Pedagogica y Tecnologica de Colombia, Facultad de Ingenieria, que dentro del grupo de investigación Grinfravial, junto con recursos propios, hizo posible el desarrollo de la investigación.

\section{REFERENCIAS}

[1] A. Shah, Q. Ali, B. Alam, K. Shahzada et al., "Study on Performance Evaluation of Adhesive Anchors in Concrete," International Journal of Advanced Structures and Geotechnical Engineering, vol. 1, no. 2, pp. 74-78, 2012.

[2] M. Obata, M. Inoue e Y. Goto, "The failure mechanism and the pull-out strength of a bond-type anchor near a free edge," Mechanics of Materials, no. 28 , pp. 113-122, 1998. https://doi. org/10.1016/S0167-6636(97)00052-5

[3] U. Priyank y S. Kimar, "Pull-out capacity of adhesive anchors: An analytical solution," International Journal of Adhesion and Adhesives, no. 60 , pp. 54-65, 2015. https://doi. org/10.1016/j.ijadhadh.2015.03.006 
[4] L. Contrafatto y R. Cosenza, "Prediction of the pull-out strength of chemical anchors in natural stone," Frattura ed Integrità Strutturale, no. 29, pp 196-208, 2014. https://doi.org/10.3221/IGF-ESIS.29.17

[5] A. Braimah, R. Guilbeault y E. Contestabile, "Strain rate behaviour of adhesive anchors in masonry," Engineering Structures, vol. 67, pp. 96-108, 2014. https://doi.org/10.1016/j.engstruct.2014.02.018

[6] S. Yilmaz, M. A. Ozen e Y. Yardim, "Tensile behavior of post-installed chemical anchors embedded to low strength concrete," Construction and Building Materials, vol. 47, pp. 861866, 2013. https://doi.org/10.1016/j. conbuildmat.2013.05.032

[7] O. Caliskan, S. Yilmaz, H. Kaplan y N. Kirac, "Shear strength of epoxy anchors embedded into low strength concrete," Construction and Building Materials, vol. 38, pp. 723-730, 2013. https://doi.org/10.1016/j.conbuildmat.2012.09.020

[8] G. Kwon, M. D. Engelhardt y R. E. Klingner, "Behavior of post-installed shear connectors under static and fatigue loading," Journal of Constructional Steel Research, vol. 66, no. 4 pp. 532541, 2010. https://doi.org/10.1016/j. jcsr.2009.09.012

[9] M. S. Hoehier, P. Mahrenholtz y R. Eligehausen, "Behavior of anchors in concrete at seismic-relevant loading rates," $\mathrm{ACl}$ Structural Journal, vol. 108, pp. 238-247, 2011. https://doi. org/10.14359/51664259
[10] M. McVay, R. Cooky K. Krishnamurthy, "Pullout simulation of post installed chemically bonded anchors," Journal Structural Engineering, vol. 122, no. 9, pp. 1016-1024, 2016. https:// doi.org/10.1061/(asce)07339445(1996)122:9(1016)

[11] W. Dongpo, W. Dongsheng, H. Siming, Z. Jun y O. Chaojun, "Behavior of post-installed large-diameter anchors in concrete foundations," Construction and Building Materials, vol. 95, pp. 124, 2015. https://doi.org/10.1016/j. conbuildmat.2015.07.129

[12] S. Yilmaz, M. A. Ozen y Y. Yardim, "Tensile behavior of post-installed chemical anchors embedded to low strength concrete," Construction and Building Materials, vol. 47, pp. 861-866, 2013. https://doi.org/10.1016/j.conbuildmat.2013.05.032

[13] American Concrete Institute, "Manual de práctica del hormigón: Informe sobre el estado del arte de los anclajes en hormigón," ACl 355.1R-91, Estados Unidos, 1997, pp. 355.1R-1-75.

[14] Argos, "Concreto de altas resistencias," 2014. [En línea]. Disponible en: http:// http://www.argos.co/Media/Colombia/ images/Ficha\%20t\%C3\%A9cnica\%20 Concreto\%20Altas\%20Resistencias.pdf

[15] Cemex, "Concretos especiales," 2016 [En línea]. Disponible en: http://www. cemexcolombia.com/SolucionesConstructor/ConcretosEspeciales.aspx

[16] L. Rocha, “Concretos especiales en la construcción. Concretos de alta resistencia," en Compilación de artículos de 
investigación octubre 2009. México, D. C.: Departamento de Materiales UAM, 2009, pp. 19-33.

[17] A. J. Murillo y E. Y. Salamanca, "Optimización de los materiales para hormigones de alta resistencia comprendidos entre 4000 Psi (28Mpa) - 6000 Psi (42 Mpa), sin aditivos," Tesis de pregrado, Facultad de Ingeniería Civil, Universidad de la Salle, Bogotá, Colombia, 2006.

[18] Sika Colombia S.A., "Hoja técnica SikaPlast MO ®," 2015. [En línea]. Disponible en: https://col.sika. com/dms/getdocument.get/16dbbdc1-a427-359c-b51d-b902ec1bb4fb/ co-ht_SikaPlast\%20MO.pdf

[19] Icontec. NTC 673 - Concretos. Ensayo de resistencia a la compresión de especímenes Cilíndricos de concreto. Bogotá, Colombia: Instituto Colombiano de Normas Técnicas y Certificación [Icontec], 2010.

[20] J. A. Galán y J. Hamón, “Estudio de la resistencia a tracción en anclajes estructurales post instalados con adhesivo epóxico," Tesis de pregrado, Facultad de Ingeniería, Universidad Pedagógica y Tecnológica de Colombia, Tunja, Colombia, 2015.

[21] R. Cook y R. Konz, "Factors Influencing Bond Strength of adhesive Anchors," $\mathrm{ACl}$ Structural Journal, vol. 98, no. 1, pp. 76-85, 2001. https://doi.org/10.14359/10149

[22] A. Çolak, "Parametric study of factors affecting the pull-out strength of steel rods bonded into precast concrete panels," International Journal of Adhesion and Adhesives," vol. 21, no. 6, pp. 487493, 2001. https://doi.org/10.1016/ S0143-7496(01)00028-8

[23] Sika Colombia S.A., "Hoja técnica Sikadur AnchorFix-4 ®," 2013. [En línea]. Disponible en: http://col.sika.com/es/ rehabilitacion-y-reforzamiento/rehabilitar-reforzar/02a005/02a005sa01.html

[24] G. C. Mays, "Performance requirements for structural adhesives in relation to concrete strengthening," International Journal of Adhesion and Adhesives, vol. 21, no. 5, pp. 423-429, 2001. https://doi.org/10.1016/S01437496(01)00019-7

[25] Icontec. NTC 2289 - Barras corrugadas y lisas de acero de baja aleación, para refuerzo de concreto. Bogotá, CoIombia: Instituto Colombiano de Normas Técnicas y Certificación [Icontec], 2007.

[26] R. A. Cook, J. Kunz, W. Fuchs R. C. Konz, "Behavior and Design of single adhesive anchors under tensile load in uncracked concrete," $\mathrm{ACl}$ Structural Journal, vol. 95, no. 1, pp.9-26, 1998. https://doi.org/10.14359/522

[27] R. A. Cook, "Behavior of chemically bonded anchors," Journal of Structural Engineering, vol. 119, no. 9, pp. 27442762, 1993. https://doi.org/10.1061/ (ASCE)0733-9445(1993)119:9(2744)

[28] R. Eligehausen, R. A. Cook y J. Appl, "Behavior and Design of Adhesive Bonded Anchors," ACl Structural Journal, vol. 103, no. 6, pp.822-831, 2006. https://doi.org/10.14359/18234 
[29] V. Romero, "Contrastación experimental de la resistencia de anclajes epóxicos," Tesis de maestría, Facultad de Ingeniería, Ingeniería, Universidad Nacional de Colombia, Bogotá, Colombia, 2005.
[30] Ministerio de Ambiente, Vivienda y Desarrollo Territorial. Reglamento Colombiano de construcción sismo resistente. NSR-10. Bogotá, Colombia: Asociación Colombiana de Ingeniería Sísmica, 2010. 